\title{
Distribution of AMPA-type glutamate receptor subunits in the chick visual system
}

R.S. Pires and L.R.G. Britto
Departamento de Fisiologia e Biofísica, Instituto de Ciências Biomédicas, Universidade de São Paulo, 05508-900 São Paulo, SP, Brasil

\section{Correspondence \\ R.S. Pires \\ Departamento de Fisiologia \\ e Biofísica \\ Instituto de Ciências Biomédicas \\ Universidade de São Paulo \\ Av. Prof. Lineu Prestes, 1524 \\ 05508-900 São Paulo, SP \\ Brasil \\ Fax: 55 (011) 818-7426 \\ E-mail: raquel@bmb.icb1.usp.br \\ Presented at the XI Annual Meeting of the Federação de Sociedades de \\ Biologia Experimental, Caxambu, \\ MG, Brasil, August 21-24, 1996. \\ Research supported by FAPESP, CNPq, FINEP and CAPES.}

Received April 3, 1996 Accepted November 6, 1996

\begin{abstract}
Several glutamate receptor (GluR) subunits have been characterized during the past few years. In the present study, subunit-specific antisera were used to determine the distribution of the AMPA-type glutamate receptor subunits GluR1-4 in retinorecipient areas of the chick brain. Six white leghorn chicks (Gallus gallus, 7-15 days old, unknown sex) were deeply anesthetized and perfused with $4 \%$ buffered paraformaldehyde and brain sections were stained using immunoperoxidase techniques. The AMPA-type glutamate receptor subunits GluR1, GluR2/3 and GluR4 were present in several retinorecipient areas, with varying degrees of colocalization. For example, perikarya in layers 2,3 , and 5 of the optic tectum contained GluR1, whereas GluR2/3 subunits appeared mainly in neurons of layer 13. The GluR4 subunit was only detected in a few cells of the tectal layer 13. GluR1 and GluR2/3 were observed in neurons of the nucleus geniculatus lateralis ventralis, whereas GluR4 was only present in its neuropil. Somata in the accessory optic nucleus appeared to contain GluR2/3 and GluR4, whereas GluR1 was the dominant subunit in the neuropil of this nucleus. These results suggest that different subpopulations of visual neurons might express different combinations of AMPA-type GluR subunits, which in turn might generate different synaptic responses to glutamate derived from retinal ganglion cell axons.
\end{abstract}

Glutamate receptors (GluR) have been categorized into two groups called ionotropic (iGluRs) and metabotropic (mGluRs) receptors. The iGluRs comprise cation-specific ion channels and are traditionally classified into three types, mainly based on pharmacological and biophysical properties: $\alpha$-amino3-hydroxy-5-methyl-4-isoxazolepropionate (AMPA)-type receptors (composed of GluR1-4 subunits), kainate-type receptors (GluR5-7, KA1-2 subunits), and $N$-methyl-D-aspartate (NMDA)-type receptors (NMDAR1, NMDA R2A-D subunits) (1). Recent molecular stud-
Key words

- Glutamate

- Receptors

- Neurotransmitters

- Visual system

- Visual pathways ies have shown that many receptor subtypes exist in all three groups of iGluRs and that they exhibit functional heterogeneity and different patterns of expression in the nervous system. Presently, various antibodies and nucleic acid probes are available for distribution and gene expression analyses. The retinorecipient areas of the brain may represent an especially useful model to study iGluR distribution and regulation since the retinal ganglion cells projecting to these areas use glutamate as a neurotransmitter (2). In the present study, commercially available anti- 
sera against the AMPA-type glutamate receptor subunits (GluR1-4) were used to determine the distribution of subunits in the visual system of the chick.

Six 7-15-day old white leghorn chicks (Gallus gallus) were used. The animals were anesthetized with ketamine and xylazine and perfused through the heart with phosphate buffered saline and 4\% paraformaldehyde in $0.1 \mathrm{M}$ sodium phosphate buffer (PB), $\mathrm{pH}$ 7.4. After $3-5 \mathrm{~h}$ of postfixation, the brains were transferred to $30 \%(\mathrm{w} / \mathrm{v})$ sucrose in PB to ensure cryoprotection. Coronal sections $(30 \mu \mathrm{m})$ of the frozen brains were cut with a sliding microtome. The free-floating brain sections were incubated with rabbit antisera against GluR1, GluR2/3 and GluR4 subunits (Chemicon, Temecula, CA) diluted 1:1001:250 in PB containing $0.3 \%$ Triton X-100 for $14-18 \mathrm{~h}$ at $4^{\circ} \mathrm{C}$. After washing 3 times $(15$ min each) with $\mathrm{PB}$, the sections were incubated with a biotinylated goat anti-rabbit antiserum diluted 1:200 in PB for $1 \mathrm{~h}$ at room temperature, and then washed with $\mathrm{PB}$ and incubated with an avidin-biotin-peroxidase complex (ABC Elite, Vector Labs., Burlingame, CA). Following the reaction with $0.05 \%$ 3-3'-diaminobenzidine and a $0.01 \%$ hydrogen peroxide in PB and intensification with $0.05 \%$ osmium tetroxide in water, the sections were mounted on gelatin and chromoalumen-coated slides, dehydrated, cleared, and coverslipped with Permount. The intensity of AMPA-type subunit immunoreactivity in the neuropil of retinorecipient areas of the chick brain was subjectively rated from absent to intense. A similar scale was used to rate the number of stained neurons. Figure 1 illustrates examples of immunohistochemical staining that were rated according to this subjective scaling. No attempt was made to quantify the intensity of staining, but only to determine the number of somata that were stained well above the background.

Moderate to intense perikaryon staining for GluR1-like immunoreactivity was ob- served in the nucleus geniculatus lateralis ventralis, nucleus lateralis anterior, nucleus lentiformis mesencephali, layers 2, 3, and 5 of the optic tectum, and the nucleus suprachiasmaticus. On the other hand, neuropil staining for the same subunit was moderate to intense in the ventral geniculate complex, griseum tecti, nucleus lentiformis mesencephali, nucleus of the basal optic root, layers 2, 3, and 5 of the optic tectum, and nucleus suprachiasmaticus. Perikarya exhibiting GluR2/3 immunoreactivity were moderate to intense in the nucleus geniculatus lateralis ventralis, nucleus of the basal optic root, layers 10 and 13 of the optic tectum, nucleus opticus principalis thalami, and nucleus suprachiasmaticus. Moderate to intense neuropil staining for GluR2/3 was observed in the griseum tecti, layers 3 and 13 of the optic tectum, nucleus opticus principalis thalami, and nucleus suprachiasmaticus. GluR4 immunoreactivity was much less frequent in perikarya than GluR1 and GluR2/3 immunoreactivities. Indeed, moderate GluR4 immunoreactivity was only observed in perikarya of the nucleus of the basal optic root. Neuropil staining for GluR4 was moderate to intense in the ventral geniculate complex, griseum tecti and the nucleus lentiformis mesencephali.

The GluR subunit immunoreactivities were differentially distributed within retinorecipient areas of the chick brain. A few structures contained all three subunits studied here. This was the case for the ventral geniculate complex, griseum tecti, nucleus lentiformis mesencephali, and the nucleus of the basal optic root. Two of three AMPAtype receptor subunits were present in the intergeniculate leaflet, nucleus lateralis anterior, layers 5-10, and 13 of the optic tectum, and nucleus suprachiasmaticus. Other structures seemed to contain only one of the glutamate receptor subunits. This pattern was observed for the area pretectalis, layers 11 and 12 of the optic tectum, and the nucleus opticus principalis thalami. No staining was 


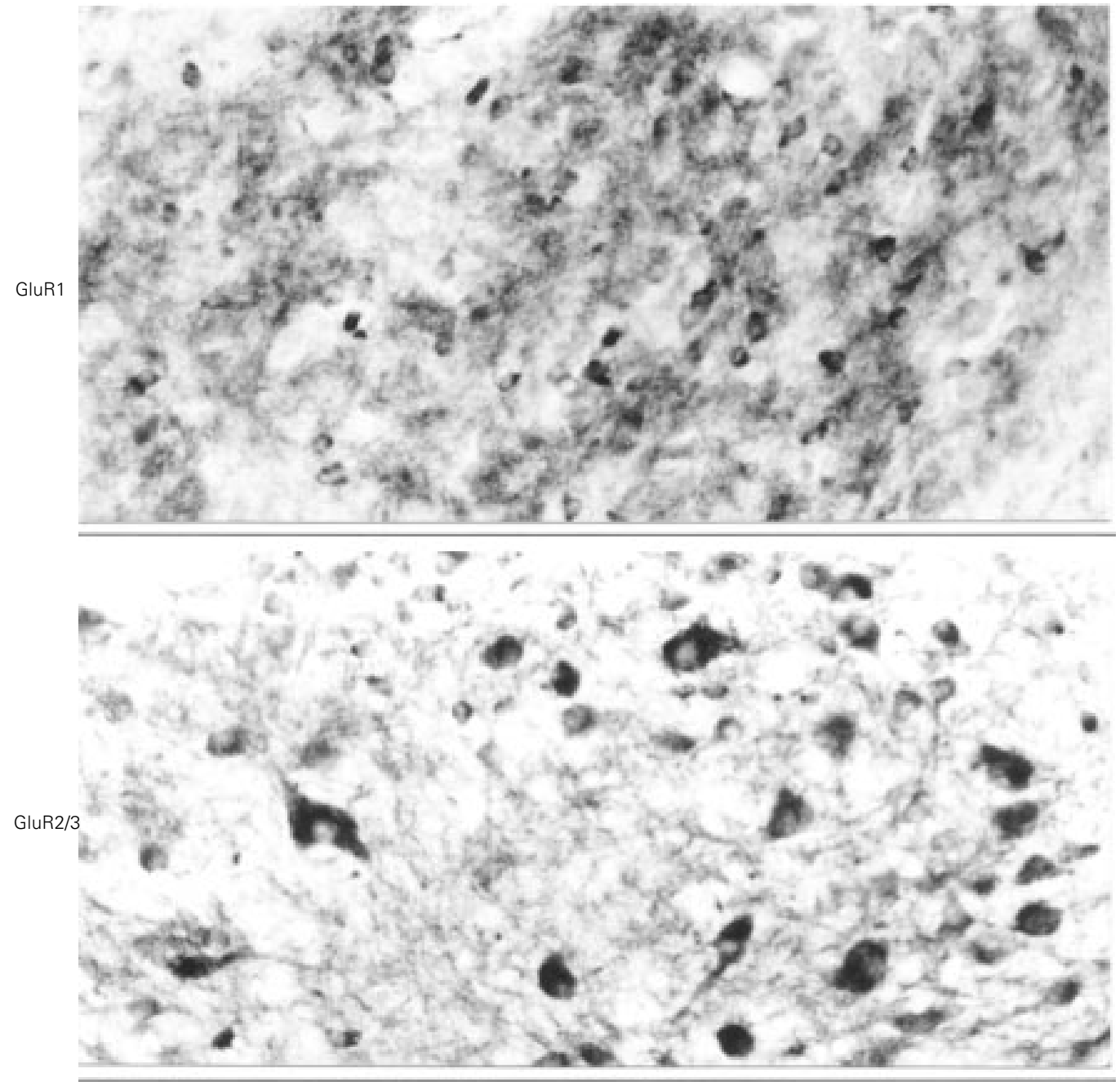

Figure 1 - Photomicrographs of coronal sections of the chick brain at the level of the nucleus of the basal optic root, processed for GluR1, GluR2/3, and GluR4 immunoreactivity. Neuropil staining is intense for GluR1, and slight for GluR2/3 and GluR4. Perikarya staining is slight for GluR1, and moderate for GluR2/3 and GluR4. Scale bar $=40 \mu \mathrm{m}$.

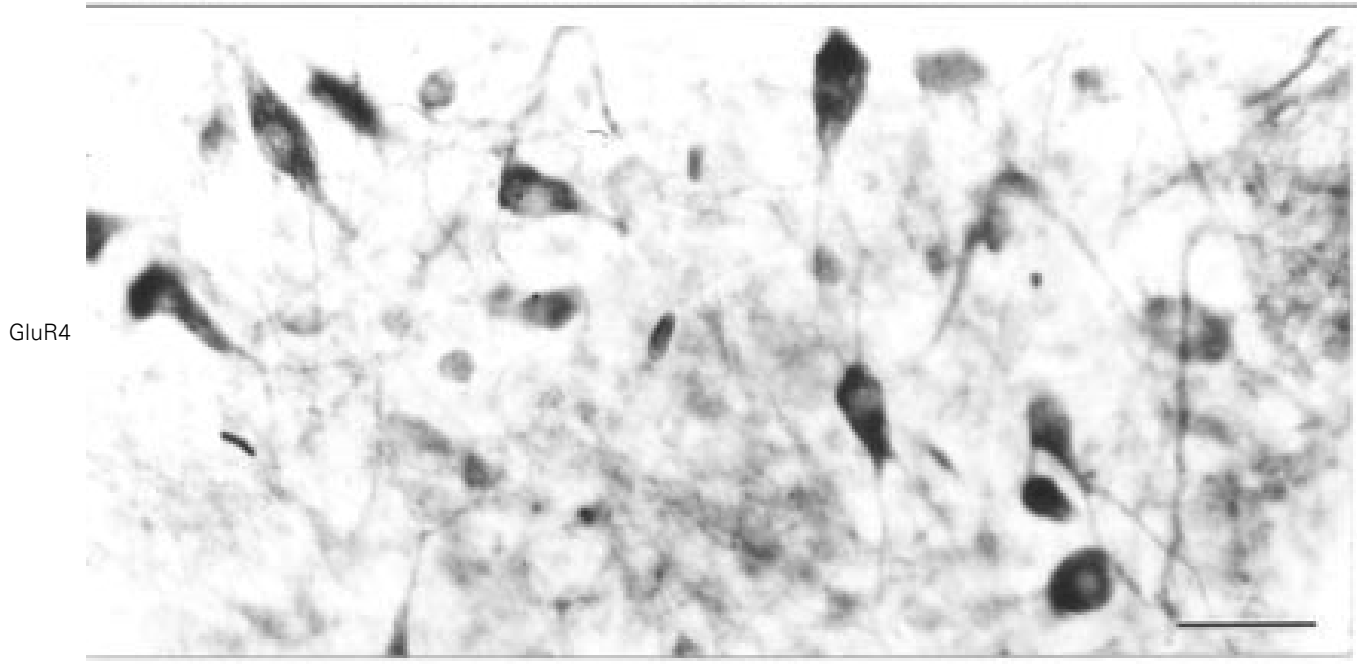


detected in layers 1,14 , and 15 of the optic tectum, and in the nucleus pretectalis diffusus. Table 1 shows a detailed account of the distribution of GluR subunits in the perikarya and neuropil of retinorecipient areas of the chick brain.

Previous immunohistochemical studies have shown the distribution of AMPA-type glutamate receptor subunits throughout the rat CNS. These studies did not focus on the localization of these subunits in the visual system. Indeed, there is little information concerning the distribution of GluRs in the visual system of other species. We found a moderate to intense staining for all AMPA subunits in the nucleus geniculatus lateralis ventralis. Petralia and Wenthold (3) noted a moderate immunoreactivity with antisera against the same AMPA-type subunits in this structure of the rat brain. Mappings of the distribution of these subunits have also revealed moderate immunoreactivity of GluR1 in the neuropil of the zonal and superficial layers of the superior colliculus, and moderate to intense perikarya staining for GluR2/3 immunoreactivity in the deep layers of the same structure $(3,4)$. Several lines of evidence indicate that the rodent superior colliculus is equivalent to the avian optic tectum (5). Indeed, in the present study we found populations of neurons in layers 2,3 , and 5 of the chick optic tectum that stained moderately to intensely with antisera against GluR1. Neurons in layers 10 and 13 of the

Table 1 - Distribution of GluR subunits in the retinorecipient areas of the chick brain.

AP, Area pretectalis; GLdp, nucleus geniculatus lateralis, pars dorsalis principalis; GLv, nucleus geniculatus lateralis, pars ventralis; GT, griseum tecti; IGL, intergeniculate leaflet; LA, nucleus lateralis anterior thalami; LMmc, nucleus lentiformis mesencephali, pars magnocellularis; $\mathrm{nBOR}$, nucleus of the basal optic root; TeO, optic tectum; OPT, nucleus opticus principalis thalami; SCNI, nucleus suprachiasmaticus, pars lateralis.

\begin{tabular}{|c|c|c|c|c|c|c|c|}
\hline \multirow{2}{*}{\multicolumn{2}{|c|}{ Structures }} & \multicolumn{2}{|c|}{ GluR1 } & \multicolumn{2}{|c|}{ GluR2/3 } & \multicolumn{2}{|c|}{ GluR4 } \\
\hline & & Perikarya & Neuropil & Perikarya & Neuropil & Perikarya & Neuropil \\
\hline \multicolumn{8}{|c|}{$\mathrm{AP}$} \\
\hline \multicolumn{8}{|l|}{ GLdp } \\
\hline \multicolumn{8}{|l|}{ GLV } \\
\hline \multicolumn{8}{|l|}{$\mathrm{GT}$} \\
\hline \multicolumn{8}{|l|}{ IGL } \\
\hline \multicolumn{8}{|l|}{ LA } \\
\hline \multicolumn{8}{|c|}{ LMmc } \\
\hline \multicolumn{8}{|c|}{$\mathrm{nBOR}$} \\
\hline \multicolumn{8}{|c|}{ TeO Layer 2} \\
\hline \multicolumn{8}{|c|}{ Layer 3} \\
\hline \multicolumn{8}{|c|}{ Layer 4} \\
\hline \multicolumn{8}{|c|}{ Layer 5} \\
\hline \multicolumn{8}{|c|}{ Layer 6} \\
\hline \multicolumn{8}{|c|}{ Layer 7} \\
\hline \multicolumn{8}{|c|}{ Layer 8} \\
\hline \multicolumn{8}{|c|}{ Layer 9} \\
\hline \multicolumn{8}{|c|}{ Layer 10} \\
\hline \multicolumn{8}{|c|}{ Layer 11} \\
\hline \multicolumn{8}{|c|}{ Layer 12} \\
\hline & Layer 13 & & & & & & \\
\hline \multicolumn{8}{|c|}{ OPT } \\
\hline \multicolumn{8}{|l|}{ SCNI } \\
\hline Abser & & Sligh & & Moderate & & Intense & \\
\hline
\end{tabular}


optic tectum showed moderate to intense immunoreactivity for GluR2/3. In the rat, GluR1 was also found in the most superficial layers of the superior colliculus, whereas GluR2/3 was predominant in its deep layers $(3,4)$. This pattern is similar to that found in the chick and described above.

Recent in situ hybridization studies revealed abundant expression of GluR1-4 mRNAs in the nucleus geniculatus lateralis ventralis, and moderate expression of GluR 1 and GluR2 mRNAs in the rat superior colliculus $(6,7)$. The immunolabeling pattern of AMPA receptor subunit distribution in our study resembles that seen in these in situ hybridization mappings. A notable difference between our results and $\left[{ }^{3} \mathrm{H}\right]$-AMPA binding data obtained in rat brain is the low binding in the nucleus geniculatus lateralis ventralis $(8,9)$, as compared to the moderate to intense staining observed here and the strong hybridization signal seen in the in situ hybridization experiments (7). Perhaps this difference reflects multiple receptor populations labeled by AMPA with different affinities (10), or simply a methodological artifact.

Induced oocyte expression of AMPAtype subunits showed that each subunit has its own pharmacological and physiological properties, and that in vivo responses to glutamate may reflect the existence of multiple receptors with different combinations of shared subunits (1). For example, the $\mathrm{Ca}^{2+}$ permeability and current-voltage $(I-V)$ relation of a channel varies with the subunit composition of the receptor. Receptors containing the GluR2 subunit showed low $\mathrm{Ca}^{2+}$ permeability, a major feature of AMPA-type receptors, whereas receptors lacking the GluR2 subunit showed high $\mathrm{Ca}^{2+}$ permeability. The present study revealed that GluR2/3 subunits were present in most visual areas, although with varying expression levels. In these areas, the GluR2/3 subunits are colocalized with GluR1 and/or GluR4 subunits. If the GluR2/3 subunits comprise functional receptors with GluR1 and GluR4 in the visual system, one could expect that most of the AMPA-type GluRs in these areas would exhibit a low $\mathrm{Ca}^{2+}$ permeability. Our experiments do not permit conclusions about this question, which could be obtained by a combination of immunoprecipitation and patch-clamp experiments.

In summary, the present study revealed an extensive distribution of AMPA-type subunits in various retinorecipient areas of the chick brain, and suggests that several patterns of response to glutamate derived from retinal ganglion cells may exist in the visual system.

\section{References}

1. Sprengel R \& Seeburg PH (1995). Ionotropic glutamate receptors. In: North RA (Editor), Handbook of Receptors and Channels, "Ligand- and Voltage-Gated lon Channels". CRC Press, Boca Raton, Florida.

2. Massey SC (1990). Cell types using glutamate as neurotransmitter in the vertebrate retina. Progress in Retinal Research, 9: 399-425.

3. Petralia RS \& Wenthold RJ (1992). Light and electron immunocytochemical localization of AMPA-selective glutamate receptors in the rat brain. Journal of Comparative Neurology, 318: 329-354.
4. Martin LJ, Blackstone CD, Levey Al, Huganir RL \& Price DL (1993). AMPA glutamate receptor subunits are differentially distributed in rat brain. Neuroscience, 53: 327-358.

5. Vanegas H (1984). Comparative Neurology of the Optic Tectum. Plenum Press, New York.

6. Matute C, Nguyen Q-T \& Miledi R (1993). mRNAs coding for neurotransmitter receptors in rabbit and rat visual areas. Journal of Neuroscience Research, 35: 652-663.

7. Sato K, Kiyama H \& Tohyama M (1993). The differential expression patterns of messenger RNAs encoding non- $\mathrm{N}$-methyl-D-aspartate glutamate receptor subunits (GluR1-4) in the rat brain. Neuroscience, 52: 515-539
8. Monaghan DT, Yao D \& Cotman CW (1984). Distribution of [ $\left.{ }^{3} \mathrm{H}\right]$ AMPA binding sites in rat brain as determined by quantitative autoradiography. Brain Research, 324: 160-164.

9. Rainbow TC, Wieczorek CM \& Halpain S (1984). Quantitative autoradiography of binding sites for [ $\left.{ }^{3} \mathrm{H}\right] \mathrm{AMPA}$, a structural analogue of glutamic acid. Brain Research, 309: 173-177.

10. Olsen RW, Szamraj O \& Houser CR (1987). [3H]AMPA binding to glutamate receptor subpopulations in rat brain. Brain Research, 402: 243-254. 